\title{
Sonographic Estimation Rather Than Quantification of Fluid Status Using Inferior Vena Cava and Other Major Vessel Parameters in a Non-Bleeding Fluid Loss and Centralization Model
}

\author{
Andreas Fichtner ${ }^{1,2}$ \\ Benedikt Brunner ${ }^{3}$ \\ Enrico Kloemich ${ }^{\prime}$ \\ Thomas Grab ${ }^{4}$ \\ Thomas Pohl ${ }^{4}$ \\ Tobias Fieback ${ }^{4}$ \\ 'Department of Anesthesiology and \\ Intensive Care Medicine, University \\ Hospital Carl Gustav Carus, Technische \\ Universität Dresden, Dresden, Saxony, \\ Germany; ${ }^{2}$ Emergency Department, \\ Freiberg Hospital, Freiberg, Saxony, \\ Germany; ${ }^{3}$ University of Gothenburg, \\ Department of Marine Sciences, \\ Gothenburg, Sweden; ${ }^{4}$ Scientific Diving \\ Center, Bergakademie Freiberg, Freiberg, \\ Saxony, Germany
}

\begin{abstract}
Objective: A well-accepted step in emergency sonography is the estimation of a fluid deficit through Inferior Vena Cava (IVC) diameter variability with known cut-offs especially in bleeding. We sought to answer, whether a non-bleeding fluid deficit can be quantified through sonographic assessment of IVC diameter variability and related aortic parameters. Sport divers were used as human hypovolemic vasoconstriction models since immersion is known to cause relevant volume depletion through vasoconstriction and induced diuresis.

Materials and Methods: Forty-one sport divers performed 342 single and repetitive dives to account for intra- and interindividual variability and were assessed for inferior Vena Cava and neighboring aortic diameters as well as their cardiac/respiratory variations. Dive-related weight loss was measured together with sonographic vessel diameter changes inferior to the right atrium. Results: Highest correlation with dive-related weight loss of max. $2.9 \mathrm{~kg}$ per an average 47 minutes dive was found with $\mathrm{r}=0.34$ for the difference of IVC maximum diameter related to minimum Aortic diameter. Single or combined parameters, as well as Collapsibility Index, showed lower or no correlations. Vascular parameters were able to explain $7.5 \%$ of the variance of fluid losses, whereas interindividual effects explained $10 \%$. The remaining $82.5 \%$ is of mixed intraindividual counterregulatory effects.

Conclusion: IVC diameter changes in immersion-induced hypovolemic centralization provides qualitative information on relevant fluid loss only. Confounding factors like inter and intraindividual variability prevent a sufficient correlation for useful quantification of the experienced non-bleeding fluid deficit in the clinical setting.
\end{abstract}

Keywords: emergency sonography, fluid status, IVC-parameters, caval index

\section{Introduction}

\section{Background}

The measurements of Inferior Vena Cava diameter and several related parameters are established ${ }^{1}$ and validated in protocols of extended focused sonography to assess the volume status especially in bleeding or hemodialysis-induced fluid loss of adults and children ${ }^{2-8}$ with high inter-rater reliability. In emergency medicine, the assessment of volume status is important, especially to diagnose hypovolemic patients and to correct their volume deficit as quickly as possible before organ damage occurs, ${ }^{9}$ which becomes even more relevant during the Covid-19Pandemic.
Correspondence: Andreas Fichtner Emergency Department, Freiberg Hospital, Donatsring 20, Freiberg, 09599, Saxony, Germany

Tel +49-373I-772198

Email andreas@drfichtner.info 
The principle behind this approach is the increasing especially respiration-dependent - diameter (d) variability of the central veins inferior to the right atrium (IVC), which correlates with increasing fluid deficit of the body, but is also prone to normalization due to plasma refilling from the interstitium. ${ }^{6,7,10,11}$ Akili et al found that sonographic dIVC as a marker of acute blood loss or fluid deficit was more accurate than the shock index, serum lactate level and base deficit. ${ }^{12}$

However, not only through respiratory cycle, but also through cardiac cycle and other abdominal and chest pressure fluctuations, central venous pressure and venous pulse influence the shape and cross-section of the IVC. ${ }^{13,14}$ Diameter changes caused by breathing-related changes of intrathoracic pressure are of major influence and can even lead to a closure of the vein. ${ }^{15}$ On the other hand, cardiac dysfunction, especially tricuspid insufficiency, has a significant influence on $\mathrm{dIVC}^{16}$ and can falsify estimations on volume status due to additional distension caused by flow reduction. Further, inaccurately set measuring points can cause caliber variations depending on the height of the abdominal gate. ${ }^{17}$ If the vein is measured obliquely, the dIVC can be overestimated. Likewise, it can be underestimated if the vein is measured out of the center.

Several attempts have been made to quantify fluid status using these easily accessible parameters despite the difficulties to obtain measurements that are directly related to fluid change in humans apart from blood donation or dialysis. In recent studies, ultrasound parameters were analysed before and after a fluid loss of almost 3 liters during a marathon $\operatorname{run}^{18}$ and less than 0.5 liters during a blood donation. ${ }^{19}$ Results of this cross-sectional study revealed a difference of $20-37 \%$ in expiratory and inspiratory IVC diameters after the fluid loss in both studies, despite large differences in the amount of fluid lost. The study population was roughly standardized and without repeated measurements of the same individual after repeated fluid loss. In another study that compared dIVC in expiration before and after hemodialysis, a formula to calculate fluid loss was found, ${ }^{10}$ which revealed a $1 \%$ decrease in body weight corresponding to a $16 \%$ decrease in dIVCmax. In ventilated ICU patients, sensitivity predicting fluid responsiveness was $69 \%$ and specificity $80 \%$ from a meta-analysis of 603 patients. ${ }^{20}$ To account for individual bio characteristics, relative changes, and relations of IVC diameters to, eg, aortic diameters, have been postulated to provide higher accuracy than absolute IVC diameters alone. ${ }^{3}$ In a study on dehydrated children, ${ }^{21}$ a clear cut-off for detection of dehydration could be defined with a sensitivity of $86 \%$ and a specificity of $56 \%$.

When comparing these results, it remains unclear, whether changes in IVC diameter and related relative indices are generalizable in bleeding and non-bleeding fluid losses, such as in severe inflammation, and if the fluid deficit can be quantified using IVC measurements. Especially in sepsis with a massive need for fluid replacement and at the same time capillary leak or even Covid-19-Pneumonia, the quantification of the demand for intravascular fluid replacement to avoid hyperhydration and pulmonary edema may be crucial.

Therefore, we sought to find a model of significant non-bleeding fluid loss in humans by avoiding confounders like extraordinary exhaustion or special training status, but including intra-individual variances and different pre-existing hydration levels as observed in emergency patients. Self-Contained Underwater Breathing Apparatus (Scuba) diving can be done at almost all ages and without an exceptional physical training condition. Divers are known to lose a considerable amount of fluid per dive, mainly due to immersion-related autonomic response with increased diuresis and other effects like vasoconstriction, sweating and humidification of dry breathing gas. ${ }^{22,23}$ In case no diving accident occurs, the physiologic alterations during diving are completely reversed upon surfacing, therefore we consider diving itself as a tool for induction of a non-bleeding fluid loss and interstitial recruitment only and thus not influencing measurements taken at the surface.

\section{Aims}

Aims of this study were to find out, whether Non-bleeding fluid loss from a standard sports Scuba dive is correlated with ultrasound-derived IVC parameter changes after the dive. If so, which absolute or relative parameter bears the best correlation? Further, can changes in ultrasound-derived IVC parameters provide sufficient information for an estimation or even quantification of the experienced fluid loss and need for replacement - especially in a non-ventilated emergency patient?

\section{Materials and Methods Study Protocol}

Forty-one Scuba divers were recruited for this observational study. All underwent independent medical examination for diving fitness and gave informed consent after university ethical approval. Three hundred and forty-two 
single and repetitive dives were monitored with consecutive 684 measurements. All divers were breathing compressed air. Individual vital signs (heart rate, blood pressure) and biodata (height, weight, sex, age, food and fluid intake, exhaustion, smoking), as well as dive profiles and surface intervals, were recorded before and after each dive. Repetitive dives were considered as dives within 12 hours after the previous dive.

The study protocol for each dive was as follows:

1. The divers presented directly before the dive after undressing and emptying their bladders since urine was considered as fluid loss. From this point on, no food or fluid was consumed.

2. Body weight was taken using a Beurer BF 105 diagnostic scale, and ultrasound parameters were recorded in flat supine position.

3. After the ultrasound examination, the divers geared up and went for the non-standardized scuba dive.

4. Directly after the dive, the divers dressed off and presented again after emptying their bladders.

5. Body weight, vital signs and dive parameters were taken, ultrasound parameters were recorded in flat supine position.

6. Food and drinks were consumed from this point until the next pre-dive measurement.

The fluid loss induced through diving was measured through the difference of body weight from before to after any dive.

\section{Ultrasound Parameters}

Ultrasound frame recording was done with a curved array multi-frequency probe of Logic e (GE Healthcare) ultrasound system by an ultrasound-experienced physician under the supervision of a certified ultrasonographer (advanced European diploma). Before and after each dive, two subxiphoidal transabdominal short-axis images were recorded 3-4 $\mathrm{cm}$ below the right atrium (Figure 1) with M-Mode beams in the axis of the maximum anterior-posterior diameter change of IVC and Aorta. Reasons for this approach were, first: identical horizontal beam with anatomical probe guidance by lower rib cage, and second: avoiding diameter measurements out of midline through short axis instead of long axis vessel cut, third: M-Mode verification of stable maximum and minimum vessel diameters over time without movement artifacts. Diameter changes dependent of cardiac and resting

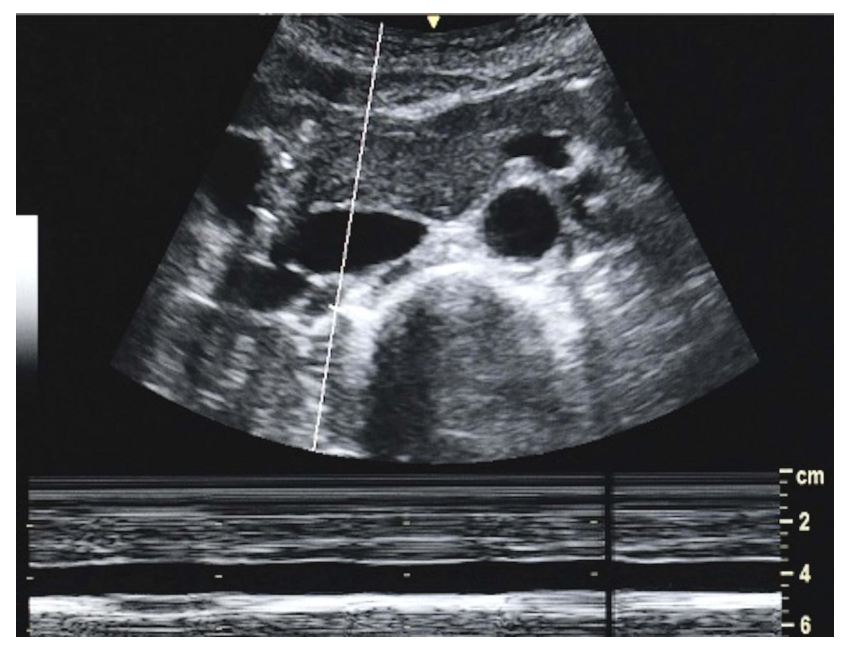

Figure I Subxiphoidal short-axis access of the major vessels for IVC (depicted) and aortic diameter measurements in the individual anterior-posterior axis of the greatest collapse. Both recordings were stored and analyzed for diameter variabilities dependent on cardiac and respiratory cycle and compared to recordings of the same diver after the measured individual weight loss in any dive after ascent.

respiratory cycles (spontaneous breathing without forced inspiration) were recorded. The rationale behind including not only respiratory cycle but also cardiac cycle dependent diameter variability assessments is the influence of possible variations in respiratory movement despite resting spontaneous breathing. Measurement parameters were minimum cardiac cycle dependent anterior-posterior IVC diameter (dIVC min) as well as minimum respiratory cycle dependent anterior-posterior IVC diameter (dIVC min insp), further maximum Inferior Vena Cava (dIVC max) and Aortic Diameters (dAo min and max), were recorded. Post- minus pre-dive differences between these parameters were calculated, as well as quotients between IVC and Aortic diameters to equalize for bio characteristics. Furthermore, IVC Collapsibility Index ((dIVCmax-min)*100/dIVCmax) was calculated using both dIVC min insp and dIVC min. These measured and calculated ultrasound parameters were then related to the measured weight loss during each dive. This was calculated for all dives, but also for the first dive of the day (no dive within the last 12 hours) and repetition dives, to account for possible influence of pre-existing dehydration or ongoing counterregulatory effects.

\section{Data and Statistical Analysis}

IVC ultrasound frames for measurements were stored as JPEG-files using individualized file names. File names were blinded and subsequently analysed by two experienced 
ultrasonographers (advanced European diploma) upon completion of all data recording. The statistical analysis was conducted using R v4.0.2. All correlations between vascular measurement results and weight losses after any dive were calculated using a Spearman-Correlation, and afterwards separately analyzed in subgroups for the first dive of the day and repetition dives (simplified as "second dive"). A mixed linear model, using the R-Package lmerTest, ${ }^{24}$ was calculated to account for the repeated measures. The marginal $\mathrm{R}^{2}$ (coefficient of determination of the fixed effects) and the conditional $\mathrm{R}^{2}$ (coefficient of determination of the fixed and the random effects) were calculated, whereas the first is comparable to the classical coefficient of determination. ${ }^{25}$ The sample size calculation of sonographic IVC assessment is published in. ${ }^{26}$

\section{Results}

\section{Study Participants}

One hundred and one dives were completed by female divers $(\mathrm{n}=13)$ and 241 dives were completed by male divers $(\mathrm{n}=28)$, aged from 15 to 69 years $($ mean $=30)$ and had a range in Body Mass Index from 20 to 34 (mean = 24). They lost on average $1.09 \mathrm{~kg}$ ( $\mathrm{sd}=0.6 \mathrm{~kg}$, maximum $2.9 \mathrm{~kg}$ ) during the dive, whereas the loss in the first dive of the day $(\mathrm{M}=1.17 \mathrm{~kg}, \mathrm{sd}=0.59 \mathrm{~kg})$ was significantly higher than in the second dive of the day $(M=0.93 \mathrm{~kg}$, $\mathrm{sd}=0.59 \mathrm{~kg} ; t$-test, $\mathrm{t}(309)=3.39, \mathrm{p}<0.001)$. The average dive time was 46 minutes ( $\mathrm{sd}=10 \mathrm{~min}$ ).

\section{Effects on Vascular Parameters}

The ultrasound-derived vascular parameters were compared before and after the first dive with a paired $t$-test (Figure 2) in the same individuals, showing significant differences (with Bonferroni adjusted $\mathrm{p}$-values) in the parameters heart rate ( $\mathrm{t}$ $(188)=-2.41, \mathrm{p}=0.017)$, systolic blood pressure $(\mathrm{t}(129)=$ $-3.44, \mathrm{p}=0.001)$, maximum IVC diameter $(\mathrm{t}(152)=-2.42$, $\mathrm{p}=0.016)$ and minimal IVC diameter $(\mathrm{t}(161)=-1.99$, $\mathrm{p}=0.0481$ ), however no difference was found for diastolic blood pressure $(\mathrm{t}(130)=0.51, \mathrm{p}=0.613)$, maximum Aortic diameter $(\mathrm{t}(202)=-1.22, \mathrm{p}=0.225)$ and minimum Aortic diameter $(\mathrm{t}(193)=-0.06, \mathrm{p}=0.955)$.

As the relative weight loss was higher for first dives compared to repetition dives, additional separate calculations for correlation were done for both types of diving (Table 1). For all single post-dive measurements (both dives, first dive, second dive), no significant correlation was found for any parameter (Heart Rate, BP Syst, BP Diast, dIVCmax,

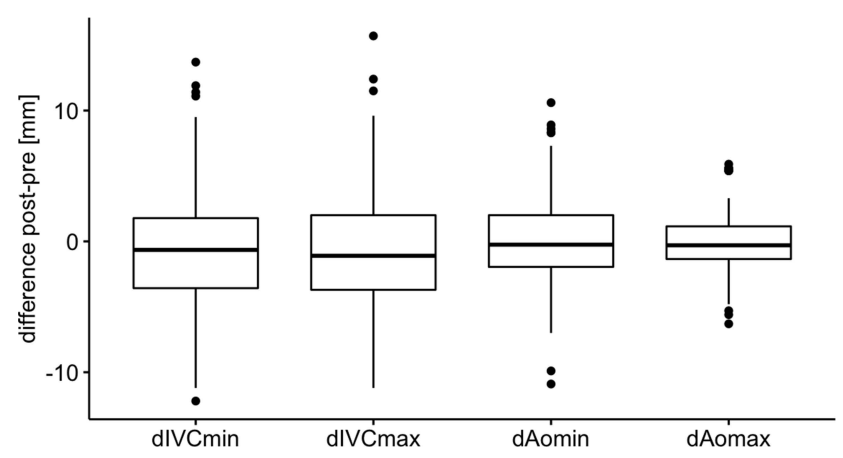

Figure 2 Comparison of measured core-parameters of the same individuals from before to after any dive. Plots are displaying median (bold horizontal line), inner quartiles $(3,4$; box, thin horizontal lines) and outer quartiles $(1,4)$ with minimum and maximum as whiskers. Outliers (deviation from inner quartile of more than 1.5 times the inner quartile range) are shown as black dots. dIVCmin insp proved to be extremely dependent on respiratory efforts with a wide spread and is not displayed, numerical values are shown in Table $\mathrm{I}$.

dIVCmin, dIVC min insp, IVCmin Collapsibility Index, IVCminInsp Collapsibility Index, dAomax, dAomin, dIVCmax/dAomin, dIVCmax/dAomax, dIVCmin/dA0min, dIVCmin insp/dAomin, dIVCmin/dAomax) as shown in the supplemental material (Table S1).

Except for dIVCmax (post-pre), no differences between the vascular parameters were found between first and repetition dives (Figure 3).

We were not able to predict the relative weight loss in a post-measurement scenario using the cardiac cycle or respiratory dependent IVC collapsibility index. In a prepost-measurement scenario, the difference of the quotient of dIVCmax and dAomin $\left(\mathrm{C}\right.$, marg. $\left.\mathrm{R}^{2}=0.07\right)$ proved to be the best predictor for experienced fluid loss (Figure 4 and

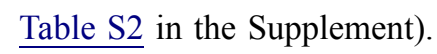

\section{Discussion}

In our model with an average weight equivalent fluid loss of more than $1 \mathrm{~kg}$ per dive with a duration of less than 1 hour, we were able to show significant changes in the minimal Aortic diameter and the minimal and maximal diameters of the Inferior Vena Cava during the cardiac cycle. We could not show weight loss related changes in the minimal diameter of the Inferior Vena Cava during respiration for a wide intraindividual variability during spontaneous breathing. Further, due to non-standardized inspiration and thus, movement of the sonographic window, many IVC inspiration measurements had to be excluded through post-hoc blinded analysis, which might be limiting our study. When accounting for biometric variability and integrating changes of sonographically derived vascular parameters into quotients or relative relations, we were able to show significant correlations of 
Table I Mean and Standard Deviation (Sd) of Vital and Ultrasound-Derived Parameter Differences (Post Minus Pre-Dive Measurements) and Quotients (Indices) to Normalize for Biometric Variability

\begin{tabular}{|c|c|c|c|c|c|c|c|c|c|c|c|c|}
\hline \multirow[t]{2}{*}{ Variable } & \multirow[t]{2}{*}{$\mathbf{n}$} & \multirow[t]{2}{*}{ Mean } & \multirow[t]{2}{*}{ sd } & \multicolumn{2}{|c|}{ All Dives } & \multirow[t]{2}{*}{ p corr } & \multicolumn{2}{|c|}{ First Dive } & \multirow[t]{2}{*}{ p corr } & \multicolumn{2}{|c|}{ Repetition Dives } & \multirow[t]{2}{*}{ p corr } \\
\hline & & & & $\mathbf{r}_{\mathrm{s}}$ & p-value & & $\mathbf{r}_{\mathbf{s}}$ & p-value & & $\mathbf{r}_{\mathbf{s}}$ & p-value & \\
\hline Heart Rate_diff & 294 & -3.09 & $|3.4|$ & 0.09 & 0.133 & ns & 0.02 & 0.750 & ns & 0.15 & 0.147 & ns \\
\hline BP Syst_diff & 197 & -3.07 & 15.44 & -0.01 & 0.917 & ns & 0.08 & 0.388 & ns & -0.09 & 0.510 & ns \\
\hline BP Diast_diff & 198 & 1.80 & 14.5 & -0.43 & 0.558 & ns & -0.015 & 0.864 & ns & -0.07 & 0.574 & ns \\
\hline dIVCmax_diff & 236 & -0.39 & 4.76 & $-0.20 *$ & 0.002 & 0.021 & -0.13 & 0.127 & ns & -0.29 & 0.012 & ns \\
\hline dIVCmin_diff & 248 & -0.42 & 4.71 & $-0.19 *$ & 0.004 & 0.042 & -0.17 & 0.031 & ns & -0.21 & 0.065 & ns \\
\hline dAomax_diff & 301 & -0.07 & 2.16 & 0.09 & 0.136 & ns & 0.11 & 0.117 & ns & 0.08 & 0.472 & ns \\
\hline dAomin_diff & 286 & 0.17 & 3.39 & 0.14 & 0.019 & ns & $0.22 *$ & 0.003 & 0.034 & 0.05 & 0.642 & ns \\
\hline dIVCmax/dAomin_diff & 204 & -0.03 & 0.45 & $-0.34 *$ & 0.000 & 0.000 & $-0.32 *$ & 0.000 & 0.000 & $-0.38 *$ & 0.001 & 0.014 \\
\hline dIVCmax/dAomax_diff & 225 & -0.01 & 0.31 & $-0.22 *$ & 0.001 & 0.013 & -0.17 & 0.042 & ns & -0.27 & 0.020 & ns \\
\hline dIVCmin/dAomin_diff & 215 & -0.06 & 0.41 & $-0.30 *$ & 0.000 & 0.002 & $-0.31 *$ & 0.000 & 0.003 & -0.22 & 0.063 & ns \\
\hline dIVCmin (insp)/dAomin_diff & 19 & 0.081 & 0.332 & 0.31 & 0.198 & ns & 0.35 & 0.201 & ns & 0.40 & 0.750 & ns \\
\hline dIVCmin/dAomax_diff & 237 & -0.03 & 0.32 & $-0.20 *$ & 0.002 & 0.041 & -0.19 & 0.017 & ns & -0.20 & 0.089 & ns \\
\hline
\end{tabular}

Notes: Spearman correlation coefficient $\left(r_{s}\right)$ with relative weight loss (percent body weight) with each the given parameters with $p$-value and Bonferroni-corrected $p$-value ( $\mathrm{p}$ corr) are calculated for I. all dives, 2 . only the first dive of the day, and 3. only dives following the first dive (repetition dives). Different numbers of datapoints ( $\mathrm{n}$ ) are due to ultrasound scans that were excluded in post-hoc analysis due to inadequate quality. The post-pre dive difference on dIVCmin in inspiration is not displayed since inspiratory efforts and their influence on IVC diameter were too variable. Correlation coefficients with a corrected $p$-value lower than 0.05 are bold and flagged with “*”.

relative weight loss (percent of body weight) with changes in post-pre dive differences of dIVCmax/dAomin, dIVCmax/ dAomax, dIVCmin(insp)/dAomin and dIVCmin/dAomax, but not cardiac- or respiratory dependent IVC Collapsibility Index ((dIVCmax-min)*100/dIVCmax). We found a maximum correlation with relative weight loss of $r=0.34$ for the post-pre difference of dIVCmax/dAomin.

Aiming to find an equation to estimate weight loss with post-pre differences, intra- and interindividually independent $\mathrm{R}^{2}$ ranges were generated, explaining a maximum of around $7.5 \%$ for all relevant biometric variability adjusted vascular parameter changes only. Including the interindividual effect (conditional $\mathrm{R}^{2}$ ), the resulting $17.5 \%$ explanation of vascular parameter change is not sufficient either. The low Intra Class Correlation of 0.13 reveals an additional high intraindividual variance that further obscures the effect of fluid loss on ultrasound-derived IVC parameters.

This means - from an ex-post view - we cannot reliably estimate or even calculate fluid deficit from the evaluated ultrasound-derived parameters. The parameter dIVCmax (expiration) and its equations to equalize biometric variability - especially when related to Aortic diameters - seem to be the only relevant ex-post parameters for a sufficient indication of a fluid deficit in the central circulatory system of a nonventilated patient. Our parameter with the highest correlation dIVCmax/dAomin Diff post-pre corresponds in an example calculation at a value of -0.60 (mean of lower quartile) to a fluid loss of - $1.26 \%$ (95\%-prediction interval: $0.15-2.36 \%$ relative weight loss). In an $80 \mathrm{~kg}$-patient, this would lead to an uncertainty between 0.12 and 1.9 liters fluid deficit - no adequate clinical guidance for fluid replacement. Both intra- and interindividual factors - more than just different inspiratory efforts alone - generate a relevant overlay of the volume-load derived diameter variability of the Inferior Vena Cava. These effects are included in our study population since the same divers went for several dives as first or repetitive dives and showed a broad variety in intra- and interindividual weight loss as well as changes in corresponding ultrasound parameters. Thus, we can answer our research questions as follows:

1. Non-bleeding fluid loss from a standard sports Scuba dive is correlated with ultrasound-derived IVC parameter changes after the dive, especially when there is no existing physiologic reaction to previous dehydration within the past 12 hours.

2. The best correlation is found for mainly respiratory independent post-pre difference of dIVCmax/ dAomin as relative parameter.

3. The change in ultrasound-derived IVC parameters after a non-bleeding fluid loss of 1 liter in less than 1 hour in a sports diver human model cannot provide sufficient information for quantification of the existing fluid deficit.

With our results, we confirm - with an even higher correlation - the results of Ragaisyte in marathon runners ${ }^{18}$ and 

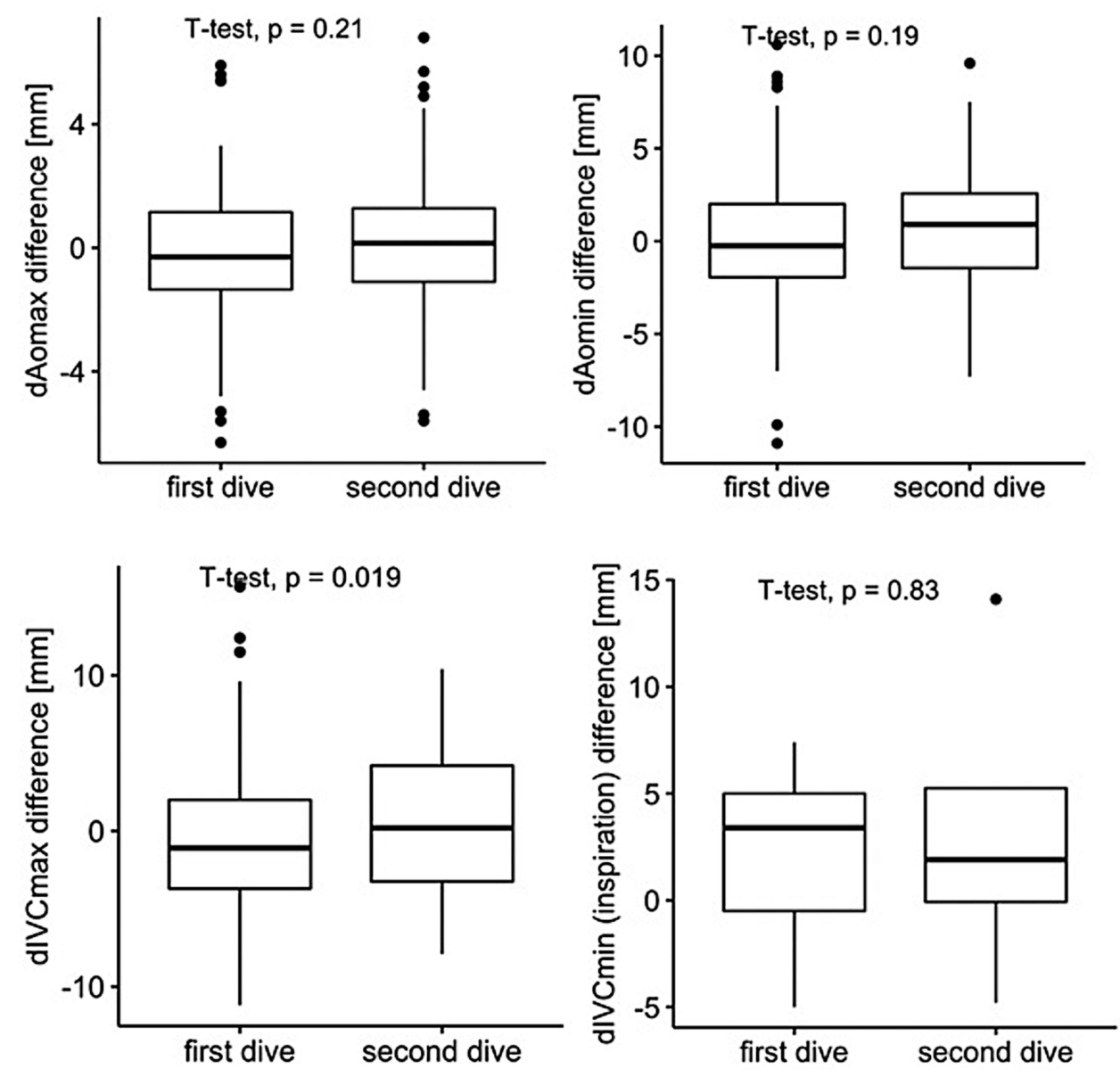

Figure 3 Differences of measured circulatory and ultrasound-derived vascular parameters after minus before any dive for the first and repetitive dive on any day. In repetitive diving, there is a trend towards smaller effect sizes of ultrasound parameters (significant difference in dIVCmax only). Plots are displaying median (bold horizontal line), inner quartiles $(3,4$; box, thin horizontal lines) and outer quartiles $(1,4)$ with minimum and maximum as whiskers. Outliers (deviation from inner quartile of more than 1.5 times the inner quartile range) are shown as black dots, numerical values in Table I.
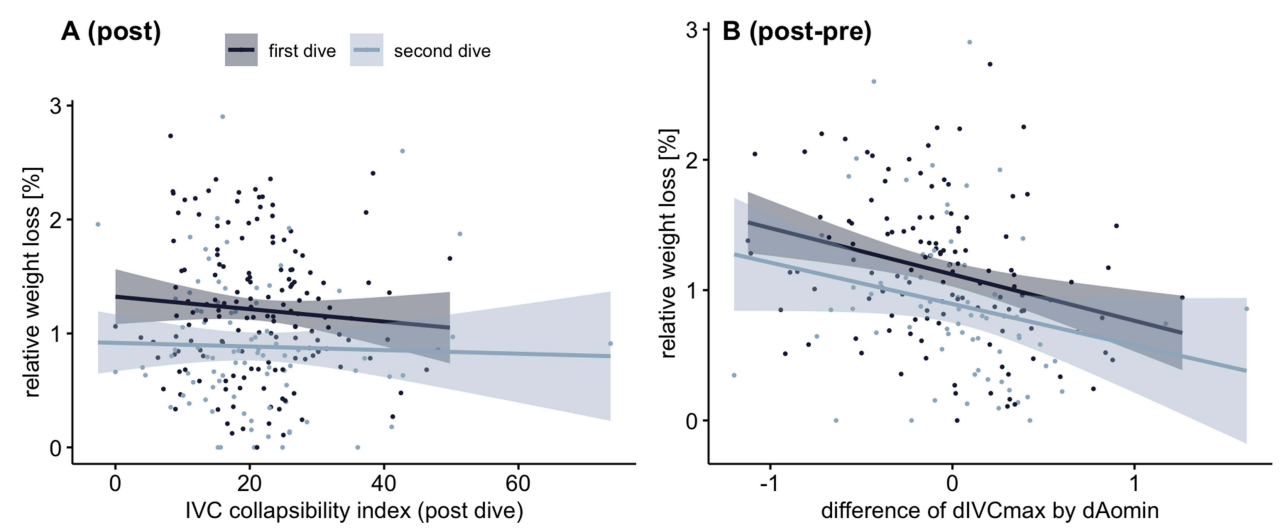

Figure 4 Scatterplots for the effect of the relative weight loss on sonographic vascular parameters, whereas the first (A) model used post dive measurements and the latter (B) post-pre dive differences. The predictions were graphically separated in measurements taken for the first dive (dark blue) and the second dive (light blue) with the confidence interval of the regression lines (shade).

Waterbrook $^{26}$ in football players that both found the best correlation of non-bleeding fluid loss to either the expiratory IVC diameter (dIVCmax) or the post-pre difference of expiratory IVC diameter related to Aortic diameter (dIVCmax/dAomin). Similar to our results, the mentioned authors found no significant correlation of the Collapsibility 
(Caval) Index. Apparent reasons for slightly differing results could be the inclusion of intraindividual variability and a broader biospectrum (BMI, age etc.) in our study population. Furthermore, athletes typically have a higher body temperature, heart rate and skin vasodilation after heavy exercise, whereas our study population experienced low physical exertion during the dives. In addition, scuba diving leads to vasoconstriction not only due to fluid loss and pressure-related effects, but also due to a cold environment with consecutive skin vasoconstriction. Therefore, our field model seems to be comparable to centralized hypovolemic Emergency Department patients when we have no information on vascular diameters and pre-existing hydration - we seek sonographic information for sufficient therapy decisions on the amount of fluid needed in Status Quo only. The condition upon presentation in the Emergency Department is typically a mixture of acute and chronic health disturbances and counter regulations. In our results, an impressive example of such an effect combination is shown by the differences between fluid loss as well as vascular diameters in repetitive diving compared to the first dive of a day. Contrary to Tetsuka et al, ${ }^{10}$ who found a linear relation of changes in dIVCmax and corresponding relative body weight after hemodialysis, Duvekot et $\mathrm{al}^{27}$ did not find a relation between total blood volume measured by radioactive isotope dilution and infusion induced acute dIVC expansion. Counterregulatory effects with participating major extracellular volume depots seem to obscure volume quantification using IVC indices.

In studies with dehydrated children, ${ }^{21,28,29}$ dIVCmax/ dAomin was also found to provide the best diameter related correlation to the effects of fluid replacement. Higher accuracy was achieved with IVCmax/Aomin relation of areas instead of diameters - both an interesting approach for further studies. The reason for this quotient being most promising compared to other parameters is that IVCmax (decreasing with fluid loss) can be assessed easily without being influenced by non-standardized ventilation effects compared to IVCmin or IVCmin (insp). Further, Aomin (but not Aomax) increases with fluid loss due to vasoconstriction and their quotient thus enhances the vascular diameter response to fluid loss for an easier qualitative recognition of a fluid deficit through the dIVCmax/ dAomin quotient in the emergency setting.

\section{Conclusion}

With our data, we can show that there are significant changes in ultrasound derived central vascular parameters after an acute non-bleeding fluid loss, so that a relevant fluid deficit can be detected in a qualitative way. However, intra- and interindividual parameters are of major influence to prevent quantification of the experienced fluid deficit from a post-hoc view in the non-ventilated human hypovolemic vasoconstriction model and even more in the emergency patient.

\section{Data Sharing Statement}

Source data is available from the corresponding author on reasonable request, detailed results are available in the Supplement Materials.

\section{Ethical Approval}

The observational study did not interfere with the diving of the participants and was conducted in accordance with the Declaration of Helsinki and approved by the university Ethics committee of TU Bergakademie Freiberg, Germany. All participants - and, in case of minors also their parents - received comprehensive information on this observational study and signed an informed consent form and agreement for publication.

\section{Acknowledgments}

We highly acknowledge the support for data acquisition by Anne Muench, Denise Preuss.

\section{Funding}

The study was supported in part by the German Society of Diving and Hyperbaric Medicine (GTUEM), and GE Healthcare regarding material provisions.

\section{Disclosure}

All authors state that they are not aware of any conflicts of interest.

\section{References}

1. Rudski LG, Lai WW, Afilalo J, et al. Guidelines for the echocardiographic assessment of the right heart in adults: a report from the American Society of Echocardiography. J Am Soc Echocardiogr. 2010;23(7):685-713. doi:10.1016/j.echo.2010.05.010

2. Mandelbaum A, Ritz E. Vena cava diameter measurement for estimation of dry weight in haemodialysis patients. Nephrol Dial Transplant. 1996;11(Suppl 2):24-27. doi:10.1093/ndt/11.supp2.24

3. Rahman NHNA, Ahmad R, Kareem MM, Mohammed MI. Ultrasonographic assessment of inferior vena cava/abdominal aorta diameter index: a new approach of assessing hypovolemic shock class 1. Int J Emerg Med. 2016;9(1):1-6. doi:10.1186/s12245-016-0101-z

4. Sönmez F, Mir S, Ozyürek AR, Cura A. The adjustment of post-dialysis dry weight based on non-invasive measurements in children. Nephrol Dial Transplant. 1996;11(8):1564-1567. doi:10. 1093/ndt/11.8.1564 
5. Kosiak W, Swieton D, Piskunowicz M. Sonographic inferior vena cava/aorta diameter index, a new approach to the body fluid status assessment in children and young adults in emergency ultrasoundpreliminary study. Am J Emerg Med. 2008;26(3):320-325. doi:10.1016/j.ajem.2007.07.012

6. Carr BG, Dean AJ, Everett WW, et al. Intensivist Bedside Ultrasound (INBU) for volume assessment in the intensive care unit: a pilot study. J Trauma. 2007;63(3):495-502.

7. Yanagawa Y, Nishi K, Sakamoto T, Okada Y. Early diagnosis of hypovolemic shock by sonographic measurement of inferior vena cava in trauma patients. $J$ Trauma. 2005;58(4):825-829. doi:10.1097/01.TA.0000145085.42116.A7

8. Dipti A, Soucy Z, Surana A, Chandra S. Role of inferior vena cava diameter in assessment of volume status: a meta-analysis. Am J Emerg Med. 2012;30(8):1414-1419.e1. doi:10.1016/j.ajem.2011.10.017

9. Padhi S, Bullock I, Li L, Stroud M; on behalf of the Guideline Development Group. Intravenous fluid therapy for adults in hospital: summary of NICE guidance. BMJ. 2013;347(dec10 1):f7073. doi:10.1136/bmj.f7073

10. Tetsuka T, Ando Y, Ono S, Asano Y. Change in inferior vena caval diameter detected by ultrasonography during and after hemodialysis. ASAIO J. 1995;41(1):105-110.

11. Barbier C, Loubières $\mathrm{Y}$, Schmit $\mathrm{C}$, et al. Respiratory changes in inferior vena cava diameter are helpful in predicting fluid responsiveness in ventilated septic patients. Intensive Care Med. 2004;30 (9):1740-1746. doi:10.1007/s00134-004-2259-8

12. Akıllı B, Bayır A, Kara F, Ak A. Inferior vena cava diameter as a marker of early hemorrhagic shock: a comparative study. Turk J Trauma. 2010;16(2):113-118.

13. Natori H, Tamaki S, Kira S. Ultrasonographic evaluation of ventilatory effect on inferior vena caval configuration. Am Rev Respir Dis. 1979;120(2):421-427.

14. Wachsberg RH. Narrowing of the upper abdominal inferior vena cava in patients with elevated intraabdominal pressure: sonographic observations. J Ultrasound Med. 2000;19(3):217-222. doi:10.7863/ jum.2000.19.3.217

15. Cheriex EC, Leunissen KML, Janssen JHA, Mooy JMV, Van Hooff JP. Echography of the inferior vena cava is a simple and reliable tool for estimation of 'dry weight'in haemodialysis patients. Nephrol Dial Transplant. 1989;4(6):563-568.

16. Sasaki T, Kubo T, Miyamoto T, Komamura K, Honda K, Miyatake K. Clinical significance of measuring inferior vena cava dimension in patients with acute exacerbation of chronic heart failure. J Cardiol. 2001;37(6):309-313.

17. Steinberg C, Weinstock DJ, Gold JP, Notterman DA. Measurements of central blood vessels in infants and children: normal values. Cathet Cardiovasc Diagn. 1992;27(3):197-201. doi:10.1002/ccd.1810270308
18. Ragaisyte E, Bardauskiene L, Zelbiene E, et al. Evaluation of volume status in a prehospital setting by ultrasonographic measurement of inferior vena cava and aorta diameters. Turk J Emerg Med. 2018;18 (4):152-157. doi:10.1016/j.tjem.2018.07.002

19. Pasquero $P$, Albani S, Sitia E, et al. Inferior vena cava diameters and collapsibility index reveal early volume depletion in a blood donor model. Crit Ultrasound J. 2015;7(1):1-7. doi:10.1186/s13089-0150034-4

20. Huang H, Shen Q, Liu Y, Xu H, Fang Y. Value of variation index of inferior vena cava diameter in predicting fluid responsiveness in patients with circulatory shock receiving mechanical ventilation: a systematic review and meta-analysis. Crit Care. 2018;22(1):204. doi:10.1186/s13054-018-2063-4

21. Chen L, Hsiao A, Langhan M, Riera A, Santucci KA. Use of bedside ultrasound to assess degree of dehydration in children with gastroenteritis. Acad Emerg Med. 2010;17(10):1042-1047. doi:10.1111/j.1553-2712.2010.00873.x

22. Pendergast DR, Moon RE, Krasney JJ, Held HE, Zamparo P. Human physiology in an aquatic environment. Compr Physiol. 2015;5 (4):1705-1750.

23. Boussuges A, Gole Y, Mourot L, et al. Haemodynamic changes after prolonged water immersion. J Sports Sci. 2009;27(6):641-649. doi:10.1080/02640410902717001

24. Kuznetsova A, Brockhoff PB, Christensen RHB. lmerTest package: tests in linear mixed effects models. J Stat Softw. 2017;82(13):1-26. doi:10.18637/jss.v082.i13

25. Zuur AF, Ieno EN, Walker N, Saveliev AA, Smith GM. Mixed effects models and extensions in ecology with R. Stat Biol Health. 2009.

26. Waterbrook AL, Shah A, Jannicky E, et al. Sonographic inferior vena cava measurements to assess hydration status in college football players during preseason camp. J Ultrasound Med. 2015;34 (2):239-245. doi:10.7863/ultra.34.2.239

27. Duvekot JJ, Cheriex EC, Tan WD, Heidendal GAK, Peeters LLH. Measurement of anterior-posterior diameter of inferior vena cava by ultrasonography: a new non-invasive method to assess acute changes in vascular filling state. Cardiovasc Res. 1994;28(8):1269-1272. doi:10.1093/cvr/28.8.1269

28. Choi YA, Kwon H, Lee JH, Jung JY, Choi YJ. Comparison of sonographic inferior vena cava and aorta indexes during fluid administered in children. Am J Emerg Med. 2018;36(9):1529-1533. doi:10.1016/j.ajem.2018.01.010

29. Chen L. Re: use of ultrasound measurement of the inferior vena cava diameter as an objective tool in the assessment of children with clinical dehydration. Acad Emerg Med. 2008;15(3):299. doi:10.11 11/j.1553-2712.2008.00057.x
Open Access Emergency Medicine

\section{Publish your work in this journal}

The Open Access Emergency Medicine is an international, peerreviewed, open access journal publishing original research, reports, editorials, reviews and commentaries on all aspects of emergency medicine. The manuscript management system is completely online and includes a very quick and fair peer-review system, which is all easy to use. Visit http://www.dovepress.com/testimonials.php to read real quotes from published authors. 